\title{
Improved approximations for ordered TSP on near-metric graphs
}

\section{Journal Article}

\section{Author(s):}

Böckenhauer, Hans-Joachim; Steinová, Monika

Publication date:

2014-12

Permanent link:

https://doi.org/10.3929/ethz-a-010887445

Rights / license:

In Copyright - Non-Commercial Use Permitted

Originally published in:

RAIRO - Theoretical Informatics and Applications 48(5), https://doi.org/10.1051/ita/2014021 


\title{
IMPROVED APPROXIMATIONS FOR ORDERED TSP ON NEAR-METRIC GRAPHS *,**
}

\author{
Hans-Joachim Böckenhauer ${ }^{1}$ And Monika Steinová ${ }^{1}$
}

\begin{abstract}
The traveling salesman problem is one of the most important problems in operations research, especially when additional precedence constraints are considered. Here, we consider the well-known variant where a linear order on $k$ special vertices is given that has to be preserved in any feasible Hamiltonian cycle. This problem is called Ordered TSP and we consider it on input instances where the edge-cost function satisfies a $\beta$-relaxed triangle inequality, i.e., where the length of a direct edge cannot exceed the cost of any detour via a third vertex by more than a factor of $\beta>1$. We design two new polynomial-time approximation algorithms for this problem. The first algorithm essentially improves over the best previously known algorithm for almost all values of $k$ and $\beta<1.087889$. The second algorithm gives a further improvement for $2 n \geq 11 k+7$ and $\beta<\sqrt[3]{4 / 3}$, where $n$ is the number of vertices in the graph.
\end{abstract}

Mathematics Subject Classification. 68Q25, 68R10.

\section{INTRODUCTION}

One of the most prominent problems in operations research is the famous Traveling Salesman Problem (TSP). This problem is studied under many generalizations and modifications [14]. It is well known that TSP is very hard to approximate, even very restricted special cases remain $\mathcal{A} \mathcal{P} \mathcal{X}$-hard. If no restrictions are given

Keywords and phrases. Approximation algorithm, near-metric ordered TSP, relaxed triangle inequality.

* This work was partially supported by SNF grant 200021-132510/1.

** An extended abstract of this work has been presented at SOFSEM 2013 [6].

1 Department of Computer Science, ETH Zurich, Switzerland.

$\{$ hjb,monika.steinova\}@inf.ethz.ch 
on the edge-cost function, the problem is not approximable by any polynomial (dependent on the input size), see for instance [15]. On the other hand, if the edge costs satisfy the standard triangle inequality, the problem becomes approximable within a factor 1.5 due to Christofides' algorithm [11]. If the metrics is even more restricted and the vertices are placed into a fixed-dimension Euclidean space, the problem turns out to have a PTAS [3]. For a brief overview of the different notions of hardness and approximability of problems, we refer, e.g., to [15].

In many applications, additional restrictions are given for the set of feasible solutions, one of the most common type of such restrictions are precedence constraints, i.e., demanding that certain vertices have to be visited before certain others. In general, such precedence constraints can be expressed by an arbitrary partial ordering on the vertices. However, in this paper, we focus on instances where a linear order on a subset of $k$ vertices is given as a part of the input and every feasible solution has to contain these special vertices in the prescribed order. We call this variant of the problem Ordered TSP or $k$-OTSP for short and its study was initialized by [8].

As we have discussed above, the properties of the edge-cost function (called $\operatorname{cost}(\cdot))$ can influence the hardness of a problem. Here, we consider the $\beta$-relaxed triangle inequality that can be formulated as $\operatorname{cost}(u v) \leq \beta(\operatorname{cost}(u w)+\operatorname{cost}(w v))$, where $u, v, w$ are arbitrary vertices of the graph and $\beta \geq 1$ is a constant. For $\beta=1$, this yields the well-known standard triangle inequality. If $\beta>1$, the inequality is referred to as $\beta$-relaxed triangle inequality. The instances where the standard or relaxed triangle inequality holds are called metric or near-metric instances, respectively. By the relaxed triangle inequality, we model how close the edgecost function is to being metric. The cases where $\beta$ is close to 1 (and hence the edge-cost function almost satisfies a triangle inequality) are most interesting for the following two reasons. First of all, many real world instances tend to have $\beta$ equal or close to 1 which supports the study of these cases. Secondly, from the theoretical viewpoint, the study of such instances allows us to better understand how the triangle inequality influences the hardness of problems and the stability of approximation algorithms $[13,15]$.

As TSP is one of the most prominent problems in complexity theory, its nearmetric instances were studied in $[1,2,5,7]$. In the metric case, the best known approximation algorithm for $k$-OTSP with approximation ratio $2.5-2 / k$ is due to $[9,10]$. In this paper, we focus on the version of $k$-OTSP where a $\beta$-relaxed triangle inequality holds which is denoted as $k-\Delta_{\beta}$ OTSP. In [8], the first polynomialtime approximation algorithm solving $k-\Delta_{\beta} \mathrm{OTSP}$ with ratio

$$
(k+1) \cdot \beta^{1+\left\lceil\log _{2}(k-1)\right\rceil} \cdot \min \left\{4 \beta, 1.5 \beta^{2}, \beta^{2}+\beta\right\}
$$

is presented. A slightly different approach was used later in [9] to improve the approximability of $k-\Delta_{\beta}$ OTSP to ratio $k \beta^{\left\lceil\log _{2}(3 k-3)\right\rceil}$ and further to $k \beta^{\left\lceil\log _{2}(\lfloor 3 k / 2\rfloor+1)\right\rceil}$ in the journal version [10]. Note that, in the original results in these papers, the ceiling function was omitted, but to be exact, the function has to be used. 
In this paper, we introduce several new observations that allow us to improve the original approximation algorithm for $k-\Delta_{\beta}$ OTSP from [8]. Our improved polynomial-time approximation algorithm yields a ratio of $3 / 2 \cdot\lceil k / 2\rceil$. $\beta^{2+\left\lceil\log _{2}(2\lceil k / 2\rceil+k-3)\right\rceil}$ which is better than the currently best known approximation algorithm for all instances with $\beta \leq(3 / 4)^{1 / \log _{2}(3 / 32)}<1.0878898$, except for those with very small $k$, see Figure 3 . The pairs $(\beta, k)$ for which we improved the approximation ratio are depicted in Figure 3. By a more involved analysis, for instances where the number of vertices is at least $5.5 k+3.5$, we achieve a further improvement even for $\beta<\sqrt[3]{4 / 3} \approx 1.12651$. We believe that some of our observations can be generalized to other problems where the $\beta$-relaxed triangle inequality holds.

The paper is organized as follows. In Section 2, we give the formal definition of the problem and fix our notation; in Section 3 we present our approximation algorithm for $k-\Delta_{\beta}$ OTSP. Section 4 discusses the more involved analysis of our algorithm and in Section 5 we conclude the paper with open problems.

\section{Preliminaries}

In this paper, we use standard notions of graph theory $[12,16]$. For an undirected graph $G=(V, E)$, the edge representation $\{u, v\}$ is simplified into an unordered pair $u v(u, v \in V)$. The set of vertices and the set of edges of a graph $G$ are denoted as $V(G)$ and $E(G)$, respectively. A list of vertices $v_{0}, \ldots, v_{n}$, where each vertex is used at most once and such that $v_{i} v_{i+1} \in E(0 \leq i<n)$, is called a path with the endpoints $v_{0}$ and $v_{n}$. The length of such path is $n$. Once the endpoints of a path are identical, i.e., $v_{0}=v_{n}$, we speak about a cycle of length $n$. A Hamiltonian cycle of a graph $G$ is a cycle of length $|V(G)|$. A graph consisting of all possible edges is referred to be complete. In a graph $G$, a subpath ${ }^{2}$ of a path $P=v_{0}, \ldots, v_{n}$ is a path $v_{i_{0}}, \ldots, v_{i_{k}}$, where $i_{0}<i_{1}<\ldots<i_{k}$ and $v_{i_{j}} v_{i_{j+1}} \in E(G)$ for all $0 \leq j<k$. We refer to the edges $v_{i_{j}} v_{i_{j+1}}$ of a subpath to be a bypass or a jump and the vertices between $v_{i_{j}}$ and $v_{i_{j+1}}$ (exclusively) in the path $P$ to be bypassed by the edge $v_{i_{j}} v_{i_{j+1}}$.

The cost of a subgraph of a graph is the sum of the costs of its edges. For simplicity, we write $\cos t(H)$ when we mean the cost of a subgraph $H$. The graphs which we refer to through out this paper are always complete and weighted.

The problem of finding a minimum-cost Hamiltonian cycle in a near-metric complete undirected graph is called Near-Metric Traveling Salesman Problem and is referred to as $\Delta_{\beta}$-TSP. If we fix, in addition, an ordered sequence of $k$ special vertices that have to be present in the minimum-cost Hamiltonian cycle in the given order, we speak about the Ordered Traveling Salesman Problem. If the underlying graph is near-metric, we refer to the problem as the Ordered Near-metric Traveling Salesman Problem and we denote it as $k-\Delta_{\beta}$ OTSP.

\footnotetext{
${ }^{2}$ Note that our notion of a subpath is a bit non-standard. The sequence of vertices in our subpath is a (not necessarily contiguous) subsequence of vertices in the path.
} 
Note that $k-\Delta_{\beta} \mathrm{OTSP}$ with $k \leq 3$ turns into the standard Near-metric Traveling Salesman Problem. Hence, in this paper, we assume that $k \geq 4$.

For the Near-metric Traveling Salesman Problem, we know three different approximation algorithms, each is best for certain values of $\beta$.

Theorem 2.1 (Andreae [1], Bender and Chekuri [5], Böckenhauer et al. [7]). There is a polynomial-time approximation algorithm solving $\Delta_{\beta}$-TSP with approximation ratio

5 Furthermore, $M=\left\{\begin{array}{lll}\frac{3}{2} \beta^{2} & \text { if } & \beta \leq 2, \\ \beta^{2}+\beta & \text { if } & 2 \leq \beta \leq 3, \\ 4 \beta & \text { if } & \beta \geq 3 .\end{array}\right.$

$$
M:=\min \left\{\frac{3}{2} \beta^{2}, \beta^{2}+\beta, 4 \beta\right\} .
$$

To be able to estimate the cost of bypasses of a path in near-metric complete graphs, we recall a very basic lemma.

Lemma 2.2 (Bandelt et al., [4]). Let $G$ be a complete graph with edge-cost function cost that satisfies the $\beta$-relaxed triangle inequality. Let $W=v_{0}, \ldots, v_{\ell}$ be a path in $G$. For $0=a_{0}<a_{1}<\ldots<a_{q}=\ell$, where $1 \leq q \leq \ell$ holds, let $m:=\max _{0 \leq i<q}\left\{a_{i+1}-a_{i}\right\}$. Then

$$
\sum_{i=0}^{q-1} \operatorname{cost}\left(v_{a_{i}}, v_{a_{i+1}}\right) \leq \beta^{\left\lceil\log _{2} m\right\rceil} \cdot \operatorname{cost}(W) .
$$

Intuitively, this lemma says that the length of a jump over a path of length $\ell$ can be bounded from above by $\beta^{\left\lceil\log _{2} \ell\right\rceil}$ times the cost of the bypassed path.

The algorithm that we present in the following section builds on the first known approximation algorithm for $k-\Delta_{\beta}$ OTSP designed by Böckenhauer et al. in [8] as Algorithm 2. Its general idea is as follows. First, connect all vertices into a Hamiltonian cycle. Secondly, color the non-special vertices clockwise as they appear in the Hamiltonian cycle cyclically by $k+1$ colors. Then connect pairs of the consecutive special vertices by clockwise traversing the Hamiltonian cycle and by including the vertices of just one fixed color (for each pair of vertices a different one) in between the two special vertices to a subpath. Use another subpath to connect the last special vertex $s_{k}$ with a vertex $x$ which was not picked to any subpath yet and is the last vertex located on the Hamiltonian cycle before the first special vertex $s_{1}$. Connect these vertices via a subpath composed of vertices of color $k$. Finally, start the last subpath in the vertex $x$ and traverse the entire cycle to the first special vertex $s_{1}$ by using the remaining vertices not yet included to the subpaths. In this paper we refer to this algorithm as HC-ALGORITHM.

A couple of years after the HC-ALGORITHM was introduced, the currently best known approximation algorithm was presented in [9] and a slightly improved version in [10]. The algorithm is based on a different underlying structure. Instead of 
a Hamiltonian cycle, a cheaper minimum spanning tree is used as an underlying structure. The vertices of the tree are colored again by $k$ colors and the special vertices are then connected by the unique paths in the tree via vertices of fixed colors. The vertices not lying on these paths are in the end attached to the cycle using some special rules and a feasible solution is obtained. In the original paper a ceiling function was omitted and hence we restate the achieved result in its correct form in the following theorem.

Theorem 2.3 (Böckenhauer et al., [10]). There exists a $k \cdot \beta^{\left\lceil\log _{2}(\lfloor 3 k / 2\rfloor+1)\right\rceil_{-a p} \text { - }}$ proximation algorithm that solves $k-\Delta_{\beta} \mathrm{OTSP}$ in polynomial time.

\section{Building ON AN UNDERLying HAMILTONiAN CYClE}

As a stepping stone to improve the approximability of $k-\Delta_{\beta} \mathrm{OTSP}(k \geq 4)$, we use the HC-Algorithm. We modify this algorithm by incorporating three new observations, which yields Algorithm 1.

The general idea of both HC-Algorithm and Algorithm 1 is the same. We first build a Hamiltonian cycle $C$ that does not respect the given order of the special vertices. The cycle is then used to construct paths between consecutive special vertices that are then merged together into a cycle where the special vertices are present in the right order. However, during the process of the path construction, some vertices might be omitted and hence are not present anymore in the new cycle. These vertices are in the end added into the cycle and the required Hamiltonian cycle is obtained.

The three observations that are improving HC-ALGORITHM are as follows.

- We can always connect three special vertices present on a cycle in the prescribed order by a single path - the path starts in the first vertex and traverses, either clockwise or counterclockwise, the cycle through the second prescribed vertex to the last vertex.

- The vertices that are bypassed by a subpath, but not included into any subpath, can be just included to the bypassing subpath. This does not increase the estimated cost of the solution as in our calculations the costs of all bypasses are estimated by the cost of the underlying path, and thus longer bypasses are always estimated as more expensive due to the relaxed triangle inequality.

- Let $H$ be a Hamiltonian cycle on a complete weighted graph and let $L_{0}, \ldots$, $L_{p-1}$ be some paths on $H$ that can be sequentially merged in their endpoints into a walk $W$, i.e., for all $0 \leq i<p$, paths $L_{i}$ and $L_{(i+1) \bmod p}$ share one endpoint. Then the vertices of $H$ that are not covered by $W$ (if there are any) form a single path $Y$ of $H$. Moreover, the adjacent vertex of an endpoint of $Y$ in $H$ is a vertex in which two paths $L_{i}$ and $L_{i+1}$ are merged. The path $Y$ split into two subpaths can be used to extend the paths $L_{i}$ and $L_{i+1}$ such that all vertices of $H$ are covered. (The situation on the path named as $L_{x}^{\prime}$ is depicted in the upper part of Fig. 2). 
Algorithm 1 (Approximation algorithm for $k-\Delta_{\beta}$ OTSP)

Input: A complete graph $G=(V, E)$ with edge cost function cost $: E \rightarrow \mathbb{Q}^{+}$that satisfies the $\beta$-relaxed triangle inequality $(\beta>1)$, and a sequence of special vertices $\left(s_{1}, \ldots, s_{k}\right)$ from $V(k \geq 4)$.

1: Using some constant-approximation algorithm, construct an approximate $\Delta_{\beta}$-TSP solution $C$ on $(G, c o s t)$, disregarding the order on $\left(s_{1}, \ldots, s_{k}\right)$.

2: Let $P$ be one of the two paths that may be obtained by removing one of the edges incident with $s_{1}$ in $C$, and let $W=\left(w_{1}, \ldots, w_{n-k}\right)$ be the sequence of the nonspecial vertices in $P$, beginning with the non-special vertex closest to $s_{1}$ in $P$. Let $f$ be a $\lceil k / 2\rceil$-cyclic coloring of non-special vertices of $C$ defined as follows: $f: V \rightarrow$ $\{-1,0, \ldots,\lceil k / 2\rceil-1\}$ where $f\left(s_{i}\right):=-1$, for all $1 \leq i \leq k$, and $f\left(w_{i+1}\right):=i \bmod$ $\lceil k / 2\rceil$, for all $0 \leq i<n-k$.

3: For $1 \leq i \leq\lceil k / 2\rceil-1$, let $L_{i-1}$ be the subpath in $C$ (either clockwise or counterclockwise) from $s_{2 i-1}$ to $s_{2 i}$ and then to $s_{2 i+1}$, restricted to $s_{2 i-1}, s_{2 i}$, and $s_{2 i+1}$ plus all vertices $w$ with $f(w)=i-1$ in between $s_{2 i-1}$ and $s_{2 i+1}$.

4: If $k$ is odd, let $L_{\lceil k / 2\rceil-1}$ be the subpath in $C$ from $s_{k}$ to $s_{1}$ that is restricted to $s_{k}, s_{1}$ and the non-special vertices $w$ that have color $f(w)=\lceil k / 2\rceil-1$.

If $k$ is even, let $L_{\lceil k / 2\rceil-1}$ be the subpath in $C$ from $s_{k-1}$ through $s_{k}$ to $s_{1}$, restricted to $s_{k-1}, s_{k}, s_{1}$ and the non-special vertices $w$ with $f(w)=\lceil k / 2\rceil-1$.

5: Create new subpaths $L_{0}^{\prime}, \ldots, L_{\lceil k / 2\rceil-1}^{\prime}$ from $L_{0}, \ldots, L_{\lceil k / 2\rceil-1}$ by including the nonspecial vertices bypassed by all $L_{i}$ as shown in Figure 1 and discussed later.

6: Let $Y$ be the path of $C$ containing all the vertices that are not included in any of the $L_{i}^{\prime}$. If such a path does not exist, skip this step. Otherwise, let $x$ and $y$ be the two endpoints of $Y$ and let $s_{2 \gamma+3}$ be the special vertex that is neighboring with $x$ in $C$ (see Fig. 2 for details).

Remove the endpoint $s_{2 \gamma+3}$ from the subpath $L_{\gamma}^{\prime}$ and extend it by including all the vertices $w$ of $Y$ in the direction from $x$ to $y$ colored by $f(w)=\gamma$ and terminate it by the vertex $y$ (if its color differs from $\gamma$ ).

Extend the subpath $L_{\gamma+1}^{\prime}$ from $s_{2 \gamma+3}$ by including all the vertices of $Y$ from $x$ to $y$ that were not included into $L_{\gamma}^{\prime}$ and terminate the modified subpath in vertex $y$.

7: Merge the subpaths $L_{0}^{\prime}, \ldots, L_{\lceil k / 2\rceil-1}^{\prime}$ into a cycle $H$.

Output The Hamiltonian cycle $H$.

Note that, in the HC-Algorithm, when the special vertices are connected by paths, the cycle is traversed always in one direction so that the special vertex $s_{1}$ is never bypassed. In our algorithm, instead, we connect an ordered triple of special vertices together in one traversal of the cycle. (If $k$ is odd, the last path is connecting just two special vertices $-s_{k}$ and $s_{1}$.) In such a traversal, we cannot always avoid the bypasses of the special vertex $s_{1}$ in the cycle.

The advantage of this approach is that connecting special vertices in triples is roughly halving the estimated cost of the solution: In our algorithm we always bound the cost of two paths connecting a pair of special vertices (except possible the last one) by the cost of the underlying structure (the cycle $C$ ). This approach of estimating the cost of more structures at once is new in this context and was 

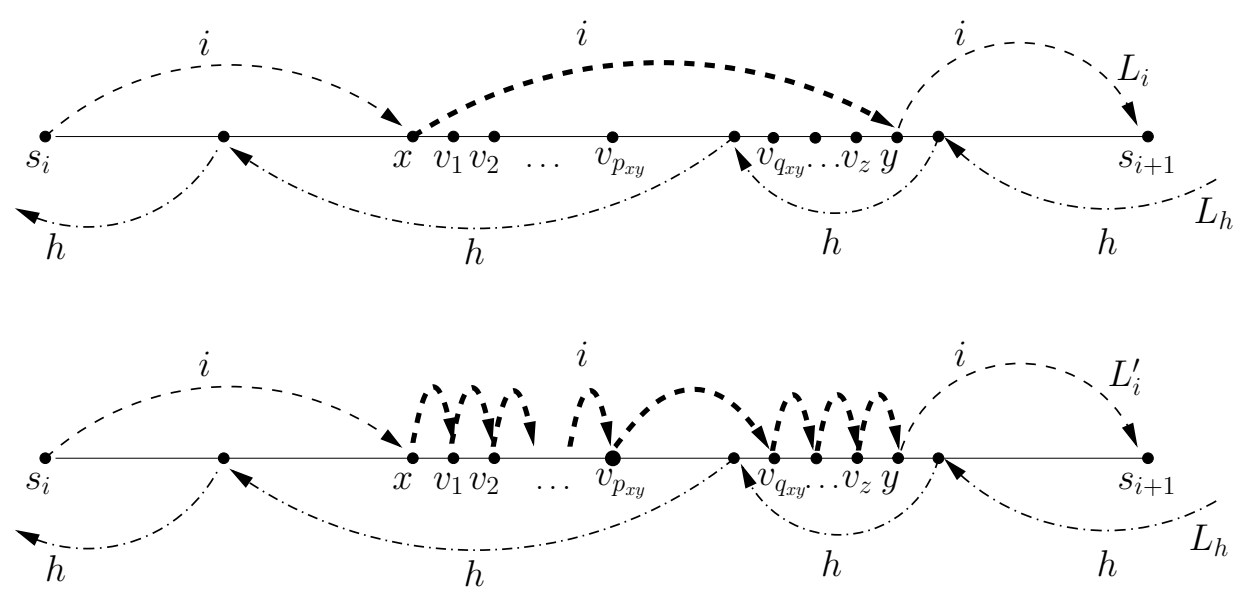

Figure 1. Modifying path $L_{i}$ to path $L_{i}^{\prime}$ : All vertices which are bypassed by the edge $x y$ of $L_{i}$ and not yet included into other subpaths are connected to subpath $L_{i}$.

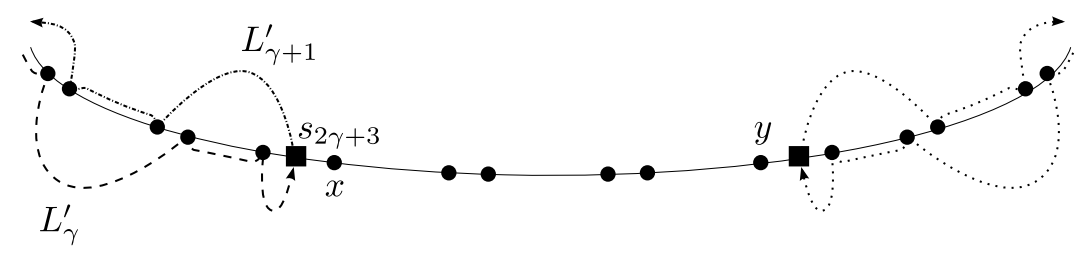

$Y$

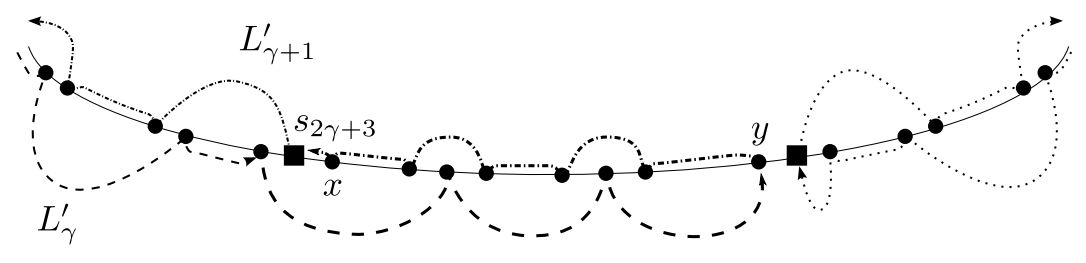

Figure 2. An extension of the paths $L_{\gamma}^{\prime}$ and $L_{\gamma+1}^{\prime}$ by the path $Y$ in Step 6 of Algorithm 1. Squares denote special vertices, dots denote non-special vertices. The terminal vertex $s_{2 \gamma+3}$ is removed from $L_{\gamma}$, the subpath $L_{\gamma}$ is extended by inclusion of all the vertices of color $\gamma$ in $Y$ and terminated by the vertex $y$. The subpath $L_{\gamma+1}^{\prime}$, in which the special vertex $s_{2 \gamma+3}$ is preserved, is extended by inclusion of all the remaining vertices of $Y$ and is terminated by the vertex $y$. 


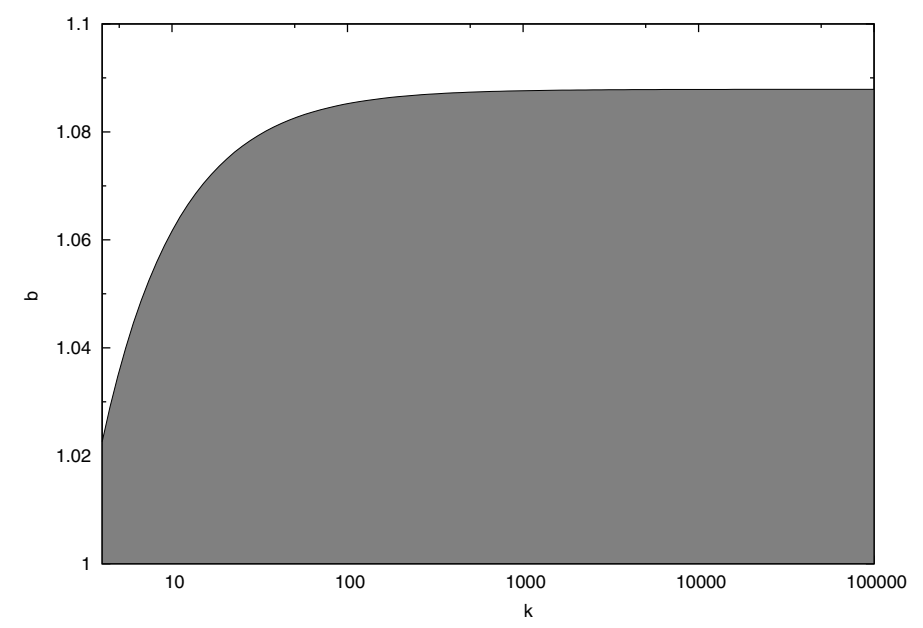

FiguRE 3 . The graph shows the pairs $(k, \beta)$ for which Algorithm 1 has provably better approximation ratio than the best known approximation algorithm.

not applied in any of the previous approximation algorithms for $k-\Delta_{\beta}$ OTSP. In all the previous approximation algorithms the cost of each path connecting a pair of special vertices was bounded by the cost of the entire underlying structure. Furthermore, the side effect of our approach is that our algorithm needs only $\lceil k / 2\rceil$ vertex-colors instead of $k+1$.

The drawback of this approach is that the bypasses might be slightly longer. More precisely, once we are passing the vertex $s_{1}$ in our traversal, we might have a cut in the coloring: the segment of the cycle between $s_{1}$ and the next vertex of the same color in the other direction than the one used for the coloring might miss vertices of some colors. This happens if the number of non-special vertices in the cycle is not divisible by the number of colors and thus the cycle does not contain the same number of vertices of each color. Here, the algorithm might be forced to bypass at most two vertices of the same color.

Lemma 3.1. In Steps 3 and 4 of Algorithm 1, at most $2\lceil k / 2\rceil+k-3$ edges of $C$ are bypassed between two consecutive vertices of subpath $L_{i}$, for all $0 \leq i \leq\lceil k / 2\rceil-1$. Furthermore, a bypass of at most $2\lceil k / 2\rceil+k-3$ edges can occur only if the vertex $s_{1}$ is bypassed. All other bypasses jump over at most $\lceil k / 2\rceil+k-2$ edges.

Proof. Let $x$ and $y$ be two consecutive vertices of subpath $L_{i}$. Since we use a cyclic coloring of the non-special vertices of $C$, the only color cut can be around the vertex $s_{1}$.

Assume that vertex $s_{1}$ is bypassed by the edge $x y$ in $L_{i}$. There are $\lceil k / 2\rceil-1$ different colors except $i$. For each such color, at most two vertices can be present in between $x$ and $y$ in $C$. In addition, there are at most $k-2$ special vertices that 
can be present here as well. Hence, the overall number of vertices between two consecutive vertices on each subpath that is bypassing the color cut is at most $2\lceil k / 2\rceil+k-4$.

Assume that vertex $s_{1}$ is not bypassed by the edge $x y$ of $L_{i}$. Then, since the coloring is cyclic, there is exactly one non-special vertex of every other color between $x$ and $y$. In addition, there are at most $k-2$ special vertices that can be present here as well. Together, there are at most $\lceil k / 2\rceil+k-3$ vertices that are bypassed by the edge $x y$, which proves the second part of the claim.

The second observation is used in Step 5 of Algorithm 1 in the same way as it was used in Step 4 of Algorithm 3 in [9] - including bypassed vertices into any subpath that is bypassing them cannot increase the cost of the subpath. The bottom line why this is so, is that the inclusion of a bypassed vertex preserves the vertex order in the subpath and does not increase the number of vertices which are bypassed. The process of the inclusion of bypassed vertices into subpaths $L_{i}$ in the construction of the subpaths $L_{i}^{\prime}$ is depicted in Figure 1 and is described in detail in [9]. From now on, we omit to write colorings and indexing modulo $\lceil k / 2\rceil$, when it is clear from the context.

Lemma 3.2. The cost of the paths $L_{i}^{\prime}$ constructed in Step 5 from paths $L_{i}$ cannot increase, i.e., for all $i, 0 \leq i \leq\lceil k / 2\rceil-1, \operatorname{cost}\left(L_{i}^{\prime}\right) \leq \operatorname{cost}\left(L_{i}\right)$.

\section{6}

At this point, if we sequentially merge the subpaths $L_{0}^{\prime}, \ldots, L_{\lceil k / 2\rceil-1}^{\prime}$ by their endpoints, we obtain a cycle $Q$ that contains each vertex at most once and that respects the given order on the special vertices. However, some vertices of $C$ might not be present in $Q$ as no subpath is bypassing them. This case is covered by the third observation - see Figure 2 for details. The vertices not included in $Q$ must form a single path $Y$ in $C$. The path $Y$ (if it exists) is the segment of the cycle $C$ through which none of the $L_{\Gamma}^{\prime}$ subpaths traversed. (Otherwise the bypassed vertices would be included in Step 5 of our algorithm and hence they would not be present in $Y$.) The vertices of $Y$ are included into two consecutive subpaths as follows. Since $Y$, with the endpoints $x$ and $y$, is a path of $C$, there must exist subpaths $L_{\gamma}^{\prime}$ and $L_{\gamma+1}^{\prime}$ with an endpoint $s_{2 \gamma+3}$ that is adjacent with $x$. We split $Y$ into two subpaths and extend each of the subpaths $L_{\gamma}^{\prime}$ and $L_{\gamma+1}^{\prime}$ by one of these split subpaths of $Y$. The outcome of these extensions is that $y$ becomes the endpoint of both subpaths and each vertex of $Y$ is included into exactly one of the extended subpaths. Notice that, in the extension of the subpath $L_{\gamma}^{\prime}$, its special vertex $s_{2 \gamma+3}$ is removed and then it is continued by inclusion of the vertices of the color $\gamma$ into the subpath up to the vertex $y$ (inclusively). Since $Y$ was not covered by $L_{\gamma}^{\prime}$, the vertices of color $\gamma$ are not already included in any subpath and this operation is sound. The other extension can be evaluated in the same way. The subpath $L_{\gamma+1}^{\prime}$ (which still contains the special vertex $s_{2 \gamma+3}$ ) is extended by including all the remaining vertices of $Y$ into the subpath and is terminated in $y$. By the same reasoning as above, this operation is also sound. For our convenience of presenting the statements comprehensively, we call the extended subpaths still $L_{\gamma}^{\prime}$ and $L_{\gamma+1}^{\prime}$. 
After the transformation of the subpaths $L_{\gamma}^{\prime}$ and $L_{\gamma+1}^{\prime}$ in Step 6, observe that two consecutive vertices in the extended subpaths are bypassing at most $2\lceil k / 2\rceil+$ $k-3$ edges in $C$. This is true as (1) the non-extended parts have this property as discussed above; (2) in the extended part of $L_{\gamma}^{\prime}$, only the vertices of color $\gamma$ are used and the distance between the last vertex of the color $\gamma$ and $y$, which may be of a different color, can be at most $2\lceil k / 2\rceil+k-3$; (3) the extended part of $L_{\gamma+1}^{\prime}$ from $s_{2 \gamma+3}$ to $y$ contains all the remaining vertices. In particular, this also includes the vertices of the color $\gamma+1$. Two consecutive vertices of this color are at distance at most $2\lceil k / 2\rceil+k-3$ in the cycle $C$. Note that this is also true for the distance between the last vertex $x$ of the extended subpath and the vertex $s_{2 \gamma+3}$. The inclusion of the vertices of the other colors in between the vertices of color $\gamma+1$ cannot increase the distance.

Lemma 3.3. The distance between two consecutive vertices in subpaths $L_{i}^{\prime}$ (for $0 \leq i \leq\lceil k / 2\rceil-1$ and $i \notin\{\gamma, \gamma+1\})$ and in the extended subpaths $L_{\gamma}^{\prime}$ and $L_{\gamma+1}^{\prime}$ is at most $2\lceil k / 2\rceil+k-3$ on the cycle $C$.

Furthermore, a bypass of at most $2\lceil k / 2\rceil+k-3$ edges can occur only if the vertex $s_{1}$ is bypassed (i.e., if the bypass occurs on the color cut). All other bypasses jump over at most $\lceil k / 2\rceil+k-2$ edges.

Proof. The lemma is proven by the discussion above and a similar reasoning as we used in Lemma 3.1.

From the description above, it is clear that the cycle $H$ computed by our algorithm is a feasible solution, i.e., each vertex of the graph is included in $H$ once and the special vertices are present in the given order. Now we are ready to estimate the approximation ratio of our algorithm.

Theorem 3.4. For $k \geq 4$, Algorithm 1 computes, in polynomial time, an approximate solution for $k-\Delta_{\beta}$ OTSP with a ratio of at most

$$
\left\lceil\frac{k}{2}\right\rceil \cdot \beta^{\left\lceil\log _{2}(2\lceil k / 2\rceil+k-3)\right\rceil} \cdot \min \left\{4 \beta, \frac{3}{2} \beta^{2}, \beta^{2}+\beta\right\} .
$$

Proof. Observe that, in a subpath $L_{i}^{\prime}$, no vertex is present multiple times. Due to the construction of the subpaths, we can always pick a path in $C$ from which we can remove vertices in contiguous blocks of bounded length to obtain the subpath $L_{i}^{\prime}$. (This is true also for the extended subpaths.) Therefore, by Lemma 3.3 we have

$$
\operatorname{cost}\left(L_{i}^{\prime}\right) \leq \beta^{\left\lceil\log _{2}(2\lceil k / 2\rceil+k-3)\right\rceil} \cdot \operatorname{cost}(C) .
$$

The cost of the merged subpaths forming the cycle $H$ can be estimated as

$$
\operatorname{cost}(H) \leq\left\lceil\frac{k}{2}\right\rceil \cdot \beta^{\left\lceil\log _{2}(2\lceil k / 2\rceil+k-3)\right\rceil} \cdot \operatorname{cost}(C) .
$$


In Step 1 of the algorithm, we use an approximation algorithm for $\Delta_{\beta}$-TSP which influences the overall ratio. The currently best approximation ratio for $\Delta_{\beta}$-TSP is

$$
\min \left\{4 \beta, \frac{3}{2} \beta^{2}, \beta^{2}+\beta\right\}
$$

and can be achieved by the best out of the currently known approximation algorithms (Thm. 2.1). This gives us the claimed ratio.

The following theorem characterizes the instances for which our algorithm improves over the $k \beta^{\left\lceil\log _{2}(\lfloor 3 k / 2\rfloor+1)\right\rceil}$-approximation algorithm of [10].

Theorem 3.5. For any $1<\beta<(3 / 4)^{\frac{1}{\log _{2} 3 / 32}}<1.08789$, there exists a fixed $k_{0} \geq 4$ such that, for all $k \geq k_{0}$, the approximation ratio of Algorithm 1 is better than the ratio achieved by Algorithm 2 of [10] for the pairs $(\beta, k)$. In these cases, the approximation ratio of Algorithm 1 is at most

$$
\left\lceil\frac{k}{2}\right\rceil \cdot \frac{3}{2} \cdot \beta^{2+\left\lceil\log _{2}(2\lceil k / 2\rceil+k-3)\right\rceil} .
$$

Proof. As the approximation algorithm for building the cycle $C$ in Step 1 of our algorithm we employ the $\frac{3}{2} \beta^{2}$-approximation algorithm for $\Delta_{\beta}$-TSP from [7]. To see for which pairs of $(k, \beta)$ our algorithm is better, we compare the approximation ratios of the two algorithms.

To simplify the calculations, we bound the estimated approximation ratio of our algorithm from above.

$$
\begin{aligned}
\frac{3}{2} \cdot\left\lceil\frac{k}{2}\right\rceil \cdot \beta^{2+\left\lceil\log _{2}(2\lceil k / 2\rceil+k-3)\right\rceil} & \leq \frac{3}{2} \cdot \frac{k+1}{2} \cdot \beta^{3+\log _{2}(k+1+k-3)} \\
& \leq \frac{3}{4} \cdot(k+1) \cdot \beta^{\log _{2}(16 k-16)}
\end{aligned}
$$

\section{6} 7 8 9

Similarly, we bound the estimated approximation ratio of the algorithm 14 from [10] from below.

$$
k \cdot \beta^{\log _{2}\left(\frac{3 k+1}{2}\right)} \leq k \cdot \beta^{\left\lceil\log _{2}(\lfloor 3 k / 2\rfloor+1)\right\rceil} .
$$

Algorithm 1 has a better approximation ratio if inequality (3.1) with the esti- 18 mated lower bound of the approximation ratio of the old $k$ - $\Delta_{\beta}$ OTSP algorithms 19 from [10] is satisfied:

$$
\frac{3}{4} \cdot(k+1) \cdot \beta^{\log _{2}(16 k-16)} \leq k \cdot \beta^{\log _{2}\left(\frac{3 k+1}{2}\right)} .
$$

After applying a couple of basic operations, the inequality can be modified into 21 its final form

$$
\log _{2} \beta \leq \frac{\log _{2} \frac{3 k+3}{4 k}}{\log _{2} \frac{3 k+1}{32 k-32}}
$$


The function on the right-hand side of inequality (3.2) for $k \geq 4$ is increasing. Hence the improvement is obtained for all $\beta$ such that

$$
\log _{2} \beta<\lim _{k \rightarrow \infty} \frac{\log _{2} \frac{3 k+3}{4 k}}{\log _{2} \frac{3 k+1}{32 k-32}}=\log _{3 / 32} \frac{3}{4} .
$$

This allows us to estimate $\beta$ to be

$$
\beta<\left(\frac{3}{4}\right)^{\frac{1}{\log _{2} 3 / 32}}<1.08788973739 .
$$

We would like to note that, at this point, we do not have to consider the other two approximation algorithms which can be used for computing the cycle $C$. Note that we achieved an improvement only for values $\beta<2$. In these cases, the $3 / 2 \beta^{2}$-approximation algorithm of Böckenhauer et al. $[7]$ is achieving the best approximation ratio out of all three approximation algorithms. Thus, the usage of the other two approximation algorithms for $k-\Delta_{\beta}$ OTSP in Step 1 of the algorithm cannot bring further improvements.

Our calculations from the proof of Theorem 3.5 imply that, for arbitrary $1<$ $\beta<\left(\frac{3}{4}\right)^{\frac{1}{\log _{2} 3 / 32}}$, there exists an initial value $k_{0} \geq 4$ such that, for all $k \geq k_{0}$, the approximation ratio of Algorithm 1 is better than the ratio of the previously best known algorithm. The pairs $(k, \beta)$ for which we obtain an improvement are depicted in Figure 3.

\section{Improving THE COLORING}

We can identify three parts of Algorithm 1 which contribute to the overall approximation ratio $3 / 2 \cdot\lceil k / 2\rceil \cdot \beta^{2+\left\lceil\log _{2}(2\lceil k / 2\rceil+k-3)\right\rceil}$. The factor $3 \beta^{2} / 2$ is given by the algorithm computing the cycle $C$ in Step 1 . The factor $\lceil k / 2\rceil$ comes from structural properties of a cycle (i.e., the number of times $\operatorname{cost}(C)$ is used to estimate the connected special vertices is halved as triples of such vertices can be connected at once). We do not aim here for the improvement of these two factors. The last part produces the factor $\beta^{\left\lceil\log _{2}(2\lceil k / 2\rceil+k-3)\right\rceil}$. Its exponent corresponds to the maximal length of a bypass in the solution and is dependent on the used vertex-coloring procedure. In this section, we introduce another observation which allows us to decrease the length of bypasses and hence to improve the approximation ratio of our algorithm.

All our bypasses in Algorithm 1 that are not bypassing the color cut around $s_{1}$ contain at most $\lceil k / 2\rceil+k-3$ vertices. Only the bypasses that occur around this cut are the bottleneck of our estimations and are increasing the estimated length of the bypasses from $\lceil k / 2\rceil+k-2$ to $2\lceil k / 2\rceil+k-3$ in all our calculations. For the ease of the presentation, we call a bypass that bypasses also vertex $s_{1}$ (and hence the color cut) a color-cut bypass. 
We estimated that each color-cut bypass contains at most $2 \cdot(\lceil k / 2\rceil-1)+x \quad 1$ vertices, where $x$ is the number of special vertices that are bypassed. Hence, the 2 main idea of the improvement is to choose a vertex from which the coloring is 3 started (and which also places the color cut into the cycle) such that each colorcut bypass contains a minimum number of special vertices.

Let $d$ be a positive integer whose precise value is specified later. Let us divide the cycle $C$ into $d$ parts of roughly the same size. From the pigeon-hole principle, one of these parts contains at most $\lfloor k / d\rfloor$ special vertices and at least $\lfloor n / d\rfloor$ of all the vertices of $C$. If this part of the cycle contains at least $3 \cdot\lceil k / 2\rceil-1$ non-special vertices, we can place the color cut into this part such that starting and ending vertices of all color-cut bypasses are present in this part. Then, the number of bypassed vertices in each color-cut bypass is at most $2 \cdot(\lceil k / 2\rceil-1)+\lfloor k / d\rfloor$. Hence, if we choose $d$ to be large (but still $d \leq n$ ), the number of vertices bypassed by each color-cut bypass is approximately $k+\frac{k}{n}$.

The last part that we have to show is that, indeed, the chosen part contains at least $3 \cdot\lceil k / 2\rceil-1$ vertices with respect to $d$ and $n$. We can estimate the number of non-special vertices in the chosen part of $C$ which is at least

$$
\left\lfloor\frac{n}{d}\right\rfloor-\left\lfloor\frac{k}{d}\right\rfloor \geq \frac{n-d+1}{d}-\frac{k}{d}=\frac{n-d+1-k}{d} .
$$

Hence, if

$$
\frac{n-d+1-k}{d} \geq 3 \cdot\left\lceil\frac{k}{2}\right\rceil-1
$$

we can place the color cut into the chosen part. The inequality above is true if

$$
\frac{n-d+1-k}{d} \geq 3 \cdot \frac{k+1}{2}-1
$$

and hence,

$$
2 \cdot(n-d+1-k) \geq 3 d \cdot(k+1)-2 d \cdot
$$

If we further modify the inequality, we are able to bound $d$ by

$$
d \leq \frac{2(n+1-k)}{3 k+3}
$$

Any positive integer value $d$ satisfying the inequality (4.1) can be used to divide the cycle into $d$ parts, one of these parts contains only $\lfloor k / d\rfloor$ special vertices which allows each color-cut bypass to contain at most $2 \cdot(\lceil k / 2\rceil-1)+\lfloor k / d\rfloor$ vertices. To minimize the length of the color-cut bypass, we need to minimize $\lfloor k / d\rfloor$ and hence $d$ has to be maximal.

Since our coloring achieves bypasses of $\lceil k / 2\rceil+k-3$ vertices for each segment of the cycle without the color cut, the partition of the cycle $C$ into many parts does not always help to decrease the length of bypasses. 
If $d=2$ (and $n \geq 4 k+2)$, the color-cut bypasses contain at most $2(\lceil k / 2\rceil-1)+$ $\lfloor k / 2\rfloor=\lceil k / 2\rceil+k-2$ vertices which is by 1 vertex more than the number of vertices bypassed in the remainder of the cycle. Therefore, if $n \geq 4 k+2$, Algorithm 1 enhanced by the special placement of the color cut has an approximation ratio of

$$
\left\lceil\frac{k}{2}\right\rceil \cdot \beta^{\left\lceil\log _{2}(\lceil k / 2\rceil+k-1)\right\rceil} \min \left\{4 \beta, \frac{3}{2} \beta^{2}, \beta^{2}+\beta\right\} .
$$

If $k \geq 4$, then $\lfloor k / 3\rfloor \leq\lfloor k / 2\rfloor-1$. Thus, if in addition $d=3$ (and hence $2 n \geq 11 k+7$ ), the color-cut bypasses become at most as long as the non-color-cut bypasses and the enhanced Algorithm 1 is achieving ratio

$$
\left\lceil\frac{k}{2}\right\rceil \cdot \beta^{\left\lceil\log _{2}(\lceil k / 2\rceil+k-2)\right\rceil} \min \left\{4 \beta, \frac{3}{2} \beta^{2}, \beta^{2}+\beta\right\} .
$$

For the latter approximation ratio with bypasses of length $\lceil k / 2\rceil+k-2$ edges, we can do similar calculations as we did in Section 3 when the $\frac{3}{2} \beta^{2}$-approximation algorithm for $\Delta_{\beta}$-TSP from Böckenhauer et al. [7] is plugged into Step 1 which yields the following theorem.

Theorem 4.1. For any $1<\beta<\sqrt[3]{\frac{4}{3}}$, there exists a fixed $k_{0} \geq 4$ such that, for all $k \geq k_{0}$ such that $2 n \geq 11 k+7$, the approximation ratio of Algorithm 1 with improved coloring is better than the ratio achieved by Algorithm 2 of [10] for the pairs $(\beta, k)$. In these cases, the approximation ratio of Algorithm 1 is at most

$$
\left\lceil\frac{k}{2}\right\rceil \cdot \frac{3}{2} \cdot \beta^{2+\left\lceil\log _{2}(\lceil k / 2\rceil+k-2)\right\rceil} .
$$

Proof. The inequality (3.1) is changed to

$$
\frac{3}{4} \cdot(k+1) \cdot \beta^{\log _{2}(12 k-12)} \leq k \cdot \beta^{\log _{2}\left(\frac{3 k+1}{2}\right)},
$$

and the inequality (3.2) changes to

$$
\log _{2} \beta \leq \frac{\log _{2} \frac{3 k+3}{4 k}}{\log _{2} \frac{3 k+1}{24 k-24}} .
$$

Again, the function on the right-hand side is increasing for $k \geq 4$ and, hence, an improvement is obtained for all $\beta$ (and all values of $k$ from some point) such that

$$
\log _{2} \beta<\lim _{k \rightarrow \infty} \frac{\log _{2} \frac{3 k+3}{4 k}}{\log _{2} \frac{3 k+1}{24 k-24}}=\log _{3 / 24} \frac{3}{4} .
$$

This allows us to estimate $\beta$ to satisfy

$$
\beta<\sqrt[3]{\frac{4}{3}}
$$

Again, as we achieved an improvement only for values $\beta<2$, there is no need to apply other two approximation algorithms for $\Delta_{\beta}$-TSP in Step 1. 


\section{Conclusion}

In this paper, we investigated approximation algorithms for the Ordered NearMetric Traveling Salesman Problem $\left(k-\Delta_{\beta}\right.$ OTSP). The first, general approximation algorithm improves the best known approximation ratio for instances with $\beta<1.087889$ and the last ratio is an improvement for $\beta<\sqrt[3]{4 / 3}$. In both cases, this is true for all $k$ except for the first finitely many small values, but, in the latter case, $k$ should be at most proportional to $n$, more precisely $2 n \geq 11 k+7$.

Even though the obtained improvement of our algorithm does not cover the entire space of instances, it broadens our knowledge on the approximability of the most interesting instances - those that are close to being metric.

There are still several open questions related to $k$ - $\Delta_{\beta}$ OTSP and $k$-OTSP. First of all, we are not aware of any hard instances for approximation algorithms which could suggest whether the estimated ratios are tight or there is still space for improvements. Furthermore, our algorithm, same as the algorithms of [8,9], is using a "static" coloring of vertices that does not reflect the actual situation in the initial Hamiltonian cycle. We believe that the approximation ratio, especially the part that is dependent on the length of bypasses, could be improved by a more sophisticated coloring.

Our enhancement from Section 4 for $n$-vertex graphs can be applied only if $n \geq 4 k+2$ (or, to be a little bit better, if $2 n \geq 11 k+7$ ), i.e., if the number of special vertices is not too high. However, it seems that the cases where $n$ and $k$ are of the same order of magnitude are the hardest ones. For these cases, one may consider an approximation algorithm that is different in its used structures from all our approaches. We can even generalize the idea and consider the problem on instances where the number of special vertices $k$ is a constant fraction of $n$.

\section{REFERENCES}

[1] T. Andreae, On the traveling salesman problem restricted to inputs satisfying a relaxed triangle inequality. Networks 38 (2001) 59-67.

[2] T. Andreae and H.-J. Bandelt, Performance guarantees for approximation algorithms depending on parametrized triangle inequalities. SIAM J. Discrete Math. 8 (1995) 1-16.

[3] S. Arora, Polynomial time approximation schemes for Euclidean traveling salesman and other geometric problems. J. ACM 45 (1998) 753-782.

[4] H.-J. Bandelt, Y. Crama and F.C.R. Spieksma, Approximation algorithms for multidimensional assignment problems with decomposable costs. Discrete Appl. Math. 49 (1994) $25-50$.

[5] M. Bender and C. Chekuri, Performance guarantees for TSP with a parametrized triangle inequality. Inf.n Proc. Lett. 73 (2000) 17-21.

[6] H.-J. Böckenhauer and M. Steinová, Improved approximations for ordered TSP on nearmetric graphs. In Proc. of the 39th International Conference on Current Trends in Theory and Practice of Computer Science (SOFSEM 2013), Vol. 7741 of Lect. Notes Comput. Sci. Springer (2013) 157-168.

[7] H.-J. Böckenhauer, J. Hromkovič, R. Klasing, S. Seibert and W. Unger, Towards the notion of stability of approximation for hard optimization tasks and the traveling salesman problem. Theor. Comput. Sci. 285 (2002) 3-24. 
[8] H.-J. Böckenhauer, J. Hromkovič, J. Kneis and J. Kupke, On the approximation hardness of some generalizations of TSP (extended abstract), in Proc. of the 10th Scandinavian Workshop on Algorithm Theory (SWAT 2006), Vol. 4059 of Lect. Notes Comput. Sci. Springer (2006) 184-195.

[9] H.-J. Böckenhauer, R. Klasing, T. Mömke and M. Steinová, Improved approximations for TSP with simple precedence constraints. In Proc. of the 7th International Conference on Algorithms and Complexity (CIAC 2010), vol. 6078 of Lect. Notes Comput. Sci. Springer (2010) 61-72.

[10] H.-J. Böckenhauer, T. Mömke and M. Steinová, Improved approximations for TSP with simple precedence constraints. J. Discrete Algorithms 21 (2013) 32-40.

[11] N. Christofides, Worst-case analysis of a new heuristic for the travelling salesman problem. Technical Report 388, Graduate School of Industrial Administration, Carnegie-Mellon University (1976).

[12] R. Diestel, Graph Theory. Springer, 3rd edition (2005).

[13] T.F. Gonzalez, Handbook of Approximation Algorithms and Metaheuristics. Chapman \& Hall/CRC Computer and Information Science Series. Chapman \& Hall/CRC (2007).

[14] G. Gutin and A.P. Punnen, The Traveling Salesman Problem and Its Variations. Combinatorial Optimization. Springer, New York (2007).

[15] J. Hromkovič, Algorithmics for Hard Problems. Introduction to Combinatorial Optimization, Randomization, Approximation, and Heuristics. Springer (2003).

[16] D.B. West, Introduction to Graph Theory. Prentice Hall Inc., Upper Saddle River, NJ, 2nd edition (2000).

Received August 6, 2013. Accepted March 31, 2014. 